\title{
Efficacy and Safety of Bi-weekly Pegfilgrastim for Dose-dense Chemotherapy-induced Neutropenia in Breast Cancer Patients
}

\author{
HITOMI MORI*, MAKOTO KUBO*, MASAYA KAI, KANAKO KURATA, \\ MAI YAMADA and MASAFUMI NAKAMURA
}

Department of Surgery and Oncology, Graduate School of Medical Sciences, Kyushu University, Fukuoka, Japan

\begin{abstract}
Background/Aim: The dose-dense doxorubicin and cyclophosphamide (ddAC) for patients with HER-2negative breast cancer is recommended by the National Comprehensive Cancer Network guideline in US. However, there are little data on serum G-CSF concentrations in patients undergoing bi-weekly dose-dense therapy with pegfilgrastim. The objective of this study was to compare the serum G-CSF concentrations in patients receiving pegfilgrastim in bi- or tri-weekly regimens. Patients and Methods: This study included 26 patients who received ddAC or docetaxel and cyclophosphamide (TC) for primary breast cancer. Serum G-CSF concentrations were measured by ELISA. Results: Serum G-CSF concentrations peaked in the second week of ddAC cases and in the ninth week of TC cases. Neutrophils gradually increased until the sixth week in ddAC cases, while they were slightly decreased during the first three weeks in TC cases. Treatments were completed without febrile neutropenia or treatment delays. Conclusion: Primary prophylactic pegfilgrastim administrations increased serum G-CSF concentrations, helping to maintain the absolute neutrophil counts that are required to undergo chemotherapy. The treatment of ddAC with $3.6 \mathrm{mg}$ pegfilgrastim is completely safe for female Japanese patients.
\end{abstract}

Granulocyte colony-stimulating factor (G-CSF) increases the absolute neutrophil count (ANC) in the blood and improves neutrophil function. Compensating for the lack of G-CSF in cancer patients experiencing neutropenia as a side effect of

This article is freely accessible online.

*These Authors contributed equally to this work.

Correspondence to: Makoto Kubo, Department of Surgery and Oncology, Graduate School of Medical Sciences, Kyushu University, 3-1-1 Maidashi Higashi-ku, Fukuoka 812-8582, Japan. Tel: +81926425441, Fax: +81926425458,e-mail: mkubo@ tumor. med.kyushu-u.ac.jp

Key Words: Pegfilgrastim, breast cancer, dose-dense, chemotherapy. chemotherapy is a crucial procedure to treat or avoid febrile neutropenia $(\mathrm{FN})$. FN increases the risk of infections and sometimes can be life-threatening. Therefore, when patients are at risk of neutropenia from chemotherapy, the risk of developing FN needs to be evaluated, and the primary prophylactic administration of G-CSF is recommended (14). Pegfilgrastim is a form of the G-CSF analog filgrastim that contains an attached polyethylene glycol (PEG). As this form stays in the body longer than filgrastim (5), it requires only one administration per cycle to manage chemotherapyinduced neutropenia. The current conventional chemotherapy regimens for breast cancer are: doxorubicin $\left(60 \mathrm{mg} / \mathrm{m}^{2}\right)$ and cyclopho-sphamide $\left(600 \mathrm{mg} / \mathrm{m}^{2} ; \mathrm{AC}\right)$, or epirubicin $\left(60 \mathrm{mg} / \mathrm{m}^{2}\right)$ and cyclophosphamide $\left(600 \mathrm{mg} / \mathrm{m}^{2}\right.$; EC) followed by docetaxel $\left(75 \mathrm{mg} / \mathrm{m}^{2} ; \mathrm{T}\right)$ every three weeks, and docetaxel $\left(75 \mathrm{mg} / \mathrm{m}^{2}\right)$ and cyclophosphamide $\left(600 \mathrm{mg} / \mathrm{m}^{2}\right.$; TC) also every three weeks. According to the recently updated clinical practice guidelines from the Japanese Society of Clinical Oncology (4), primary prophylactic pegfilgrastim administration is permitted when combined with dose-dense AC (ddAC) every two weeks, TC, docetaxel $\left(75 \mathrm{mg} / \mathrm{m}^{2}\right)$, doxorubicin $\left(50 \mathrm{mg} / \mathrm{m}^{2}\right)$ and cyclophosphamide $\left(500 \mathrm{mg} / \mathrm{m}^{2}\right.$; TAC), or 5-fluorouracil $\left(500 \mathrm{mg} / \mathrm{m}^{2}\right)$, epirubicin $\left(100 \mathrm{mg} / \mathrm{m}^{2}\right)$ and cyclophosphamide $\left(500 \mathrm{mg} / \mathrm{m}^{2}\right.$; FEC) every three weeks, as FN is expected in $>20 \%$ of these cases.

Dose-dense chemotherapy is a treatment requiring G-CSF drugs such as filgrastim or pegfilgrastim that aims to achieve higher rates of cancer cell death and has been adopted as an adjuvant therapy in high-risk breast cancer cases with the goal of improving outcomes. The number of cancer cells often rebounds during conventional chemotherapy, making it difficult for the anticancer agents to completely succeed before treatment ends. However, there is an alternative treatment approach that compresses the conventional schedule, giving the same number of doses and cycles over a shorter time period. This simple manipulation has been shown to achieve considerably greater efficacy by minimizing cancer cell regrowth between treatment cycles (6).

The polar, randomized Cancer and Leukemia Group B (CALGB) Trial 9741, which was led by Citron et al., 
demonstrated that dose-dense therapy combined with filgrastim (bi-weekly) improved both disease-free and overall survival compared with conventional therapy (tri-weekly). Dose-dense therapy significantly reduced the risks of recurrence and death by $26 \%$ and $31 \%$, respectively (7). Consistently, Del Mastro et al. reported the multicentre randomized Gruppo Italiano Mammella (GIM) 2 phase III trial, which showed that dose-dense therapy combined with pegfilgrastim significantly reduced the risks of recurrence and death by $23 \%$ and $35 \%$, respectively (8).

Meanwhile, the Phase III placebo-controlled, doubleblind, randomized trial led by Kosaka et al. demonstrated that pegfilgrastim reduced the $\mathrm{FN}$ risk in breast cancer patients receiving TC therapy (9); the $\mathrm{FN}$ incidence was $1.2 \%$ in the pegfilgrastim group compared with $68.8 \%$ in the placebo group. Patients in the pegfilgrastim group required less hospitalization and antibiotics for FN. Additionally, the latest National Comprehensive Cancer Network (NCCN) guidelines for breast cancer treatment also recommend ddAC followed by T or TC for HER-2-negative breast cancer (10). However, there is little data on the effect on serum G-CSF concentrations in Japanese patients undergoing bi-weekly dose-dense therapy with pegfilgrastim. Thus, the objective of this study was to compare serum G-CSF concentrations, $\mathrm{ANC}$, and relative dose intensity (RDI) when using pegfilgrastim bi- and tri-weekly, and to investigate whether dose-dense chemotherapy can be safely completed without $\mathrm{FN}$ in Japanese patients.

\section{Patients and Methods}

Patients. This study included 26 patients who received ddAC or TC as neoadjuvant or adjuvant chemotherapy for primary breast cancer at Kyushu University Hospital (Fukuoka, Japan) between July 2015 and September 2017. Patients received their respective treatments according to the NCCN Guidelines (10), the Clinical Practice Guideline of Breast Cancer by the Japanese Breast Cancer Society (11), or the recommendations of the St. Gallen International Breast Cancer Conference (12-15). The study conformed to the principles of the Declaration of Helsinki and was approved by the Institutional Review Board of Kyushu University Hospital (No. 29-119). Written informed consent was obtained from all patients prior to their inclusion in this study.

Treatments. Among the 26 patients, 13 received bi-weekly ddAC and 13 received tri-weekly TC. Pegfilgrastim $(3.6 \mathrm{mg})$ was injected subcutaneously once 24-72 h after chemotherapy.

Sample analysis and safety assessment. Patient blood samples (5 $\mathrm{ml})$ were collected immediately before receiving chemotherapy in every cycle to evaluate serum G-CSF concentrations. Blood samples were centrifuged at $3000 \times g$ for $5 \mathrm{~min}$ to separate serum, and the supernatant was collected and stored at $-80^{\circ} \mathrm{C}$ until further use. GCSF concentrations were measured by applying serum samples to an ELISA for human G-CSF (R\&D Systems, Minneapolis, MN, USA) at $450 \mathrm{~nm}$. A standard was included to generate a standard curve and to serve as a positive control for which the concentration was already known; absorbance measurements were performed in triplicate.

Neutrophils and lymphocytes were also counted using the same blood samples (before receiving chemotherapy in every cycle). According to Japanese Society of Medical Oncology Guidelines, FN is defined as an axillary body temperature $\geq 37.5^{\circ} \mathrm{C}$, an oral temperature $\geq 38^{\circ} \mathrm{C}$, or ANC $\leq 500 / \mu$ l or expected to fall below $\leq 500 / \mu \mathrm{l}$ within $48 \mathrm{~h}(16)$.

Statistical analysis. The RDI was calculated as follows: $\mathrm{RDI}=$ received dose intensity $\left(\mathrm{mg} / \mathrm{m}^{2} /\right.$ week $) /$ planned dose intensity $\left(\mathrm{mg} / \mathrm{m}^{2} /\right.$ week) for each chemotherapy regimen. $p$-Values $<0.05$ were considered statistically significant. Statistical analyses were performed using JMP v11 (SAS Institute Inc., Cary, NC, USA).

\section{Results}

Patients receiving ddAC were younger than those receiving TC. All patients receiving ddAC had lymph node metastases. The tumours of patients receiving ddAC included all subtypes except Luminal A, while the tumour subtypes of patients receiving TC were mostly Luminal B (Table I).

Figure 1 shows serum G-CSF concentrations as measured by ELISA. At 14 days after starting chemotherapy (one cycle), serum G-CSF concentrations had increased 9-fold on average in the ddAC group; in contrast, serum G-CSF concentrations doubled on average after one cycle (3 weeks) in the TC group. We next focused on the trend of serum G-CSF concentrations following four treatment cycles and found that the peak serum G-CSF concentrations was in the second week for ddAC cases and between 6-9 weeks for TC cases.

Figure 2 shows blood ANCs. After one therapy cycle (2 weeks) neutrophils had increased by $40 \%$ on average in ddAC cases, while in TC cases the ANC decreased by $20 \%$ on average during the first cycle (3 weeks). Extending this analysis out to four treatment cycles showed that neutrophils gradually increased over the first 6 weeks in ddAC cases but decreased slightly during the first 3 weeks of TC therapy, but then increased until the ninth week of treatment.

Among the 26 patients, 25 started chemotherapy under dose reductions of $-5 \%$ to $-20 \%$. The mean ddAC RDI was $92 \%$ and that of TC was $86 \%$ (Figure 3 ). The ddAC RDI was significantly higher than that of TC ( $p=0.0003$; Figure 4). The mean RDI tended to be lower in elderly patients $(>50$ years-old) (Figure 3). Two patients receiving ddAC and five receiving TC experienced dose reductions during treatment due to side effects such as dysgeusia (grade 2) and fatigue (grade 2). Importantly, no patients experienced FN.

Twenty of the 26 patients completed therapy without FN or treatment delay; six cases had to discontinue chemotherapy because of drug eruption, depression or progressive disease (Table II). 

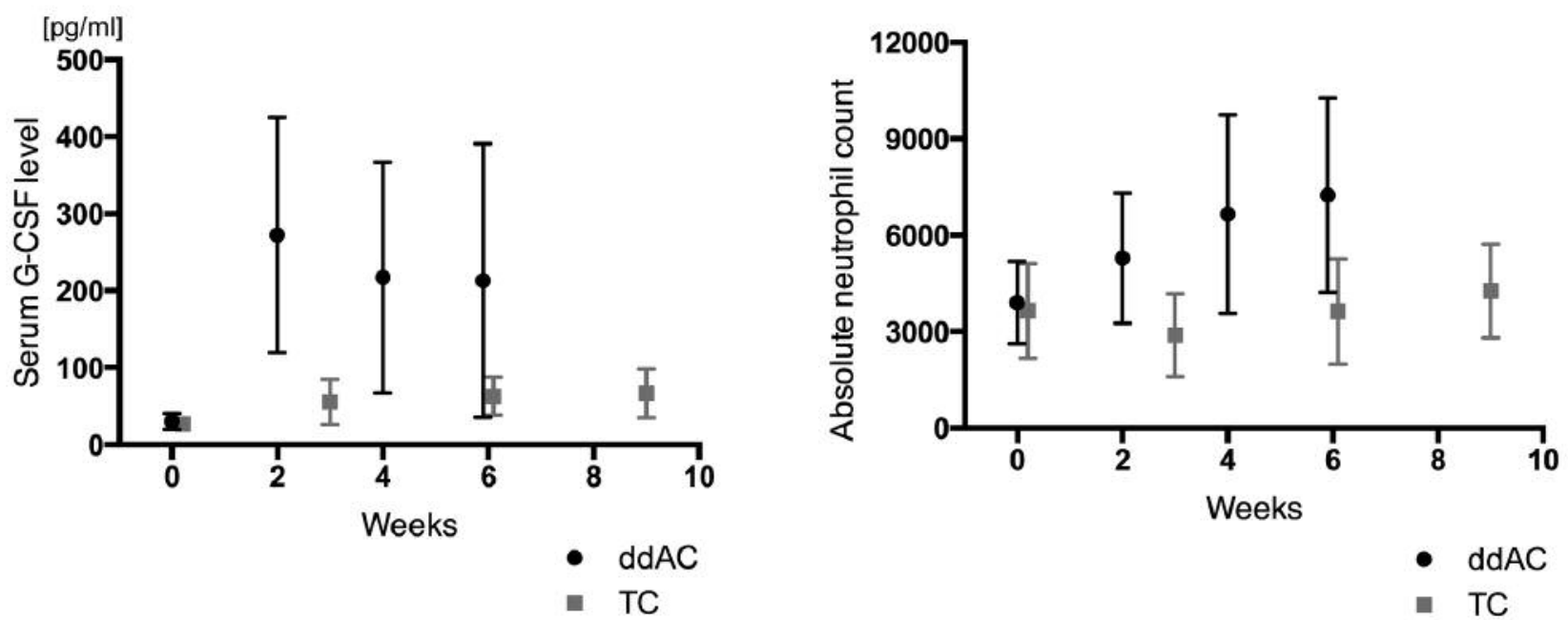

Figure 1. Average serum G-CSF concentrations of the ddAC and TC

Figure 2. Average absolute neutrophil count of the ddAC and TC cases. cases.

Table I. Patient and tumour characteristics.

\begin{tabular}{|c|c|c|c|c|c|c|}
\hline \multirow{2}{*}{ Age at diagnosis } & \multicolumn{2}{|c|}{$\begin{array}{c}\mathrm{ddAC}(\mathrm{NAC}) \\
\mathrm{N}=7\end{array}$} & \multicolumn{2}{|c|}{$\begin{array}{c}\text { ddAC (Adjuvant) } \\
\mathrm{N}=6\end{array}$} & \multicolumn{2}{|c|}{$\begin{array}{c}\text { TC (Adjuvant) } \\
\mathrm{N}=13\end{array}$} \\
\hline & & & & & & \\
\hline Mean (Range) & 45 & $(32-61)$ & 46 & $(37-57)$ & 53 & $(42-68)$ \\
\hline \multicolumn{7}{|l|}{ Tumor size } \\
\hline $\mathrm{T} 1(\leq 2 \mathrm{~cm})$ & 0 & & 2 & $(33 \%)$ & 4 & $(31 \%)$ \\
\hline $\mathrm{T} 2(>2 \mathrm{~cm}, \leq 5 \mathrm{~cm})$ & 3 & $(43 \%)$ & 3 & $(50 \%)$ & 7 & $(54 \%)$ \\
\hline $\mathrm{T} 3(>5 \mathrm{~cm})$ & 1 & $(14 \%)$ & 1 & $(17 \%)$ & 2 & $(15 \%)$ \\
\hline $\mathrm{T} 4$ & 3 & $(43 \%)$ & 0 & & 0 & $(0 \%)$ \\
\hline \multicolumn{7}{|l|}{ Node status } \\
\hline No & 0 & & 0 & & 8 & $(62 \%)$ \\
\hline N1 (Axillary) & 4 & $(57 \%)$ & 3 & $(50 \%)$ & 5 & $(38 \%)$ \\
\hline N2 (Parasternal) & 1 & $(14 \%)$ & 3 & $(50 \%)$ & 0 & \\
\hline N3 (Subclavicular) & 2 & $(29 \%)$ & 0 & & 0 & \\
\hline \multicolumn{7}{|l|}{ Subtype } \\
\hline Luminal A & 0 & & 0 & & 1 & $(8 \%)$ \\
\hline Luminal B & 3 & $(43 \%)$ & 3 & $(50 \%)$ & 12 & $(92 \%)$ \\
\hline Luminal-HER2 & 1 & $(14 \%)$ & 0 & & 0 & \\
\hline HER2 & 0 & & 2 & $(33 \%)$ & 0 & \\
\hline Triple-negative & 3 & $(43 \%)$ & 1 & $(17 \%)$ & 0 & \\
\hline
\end{tabular}

Table II. Summary of completed or discontinued treatment cycles.

\begin{tabular}{|c|c|c|c|c|c|c|}
\hline \multirow[b]{2}{*}{ Completed } & \multicolumn{2}{|c|}{$\begin{array}{c}\mathrm{ddAC}(\mathrm{NAC}) \\
\mathrm{N}=7\end{array}$} & \multicolumn{2}{|c|}{$\begin{array}{c}\text { ddAC (Adjuvant) } \\
\mathrm{N}=6\end{array}$} & \multicolumn{2}{|c|}{$\begin{array}{c}\text { TC (Adjuvant) } \\
\mathrm{N}=13\end{array}$} \\
\hline & 4 & $(57 \%)$ & 6 & $(100 \%)$ & 10 & $(77 \%)$ \\
\hline \multicolumn{7}{|l|}{ Discontinue } \\
\hline Drug eruption & 0 & & 0 & & 2 & $(15 \%)$ \\
\hline Depression & 0 & & 0 & & 1 & $(8 \%)$ \\
\hline Progressive disease & 3 & $(43 \%)$ & 0 & & 0 & \\
\hline
\end{tabular}



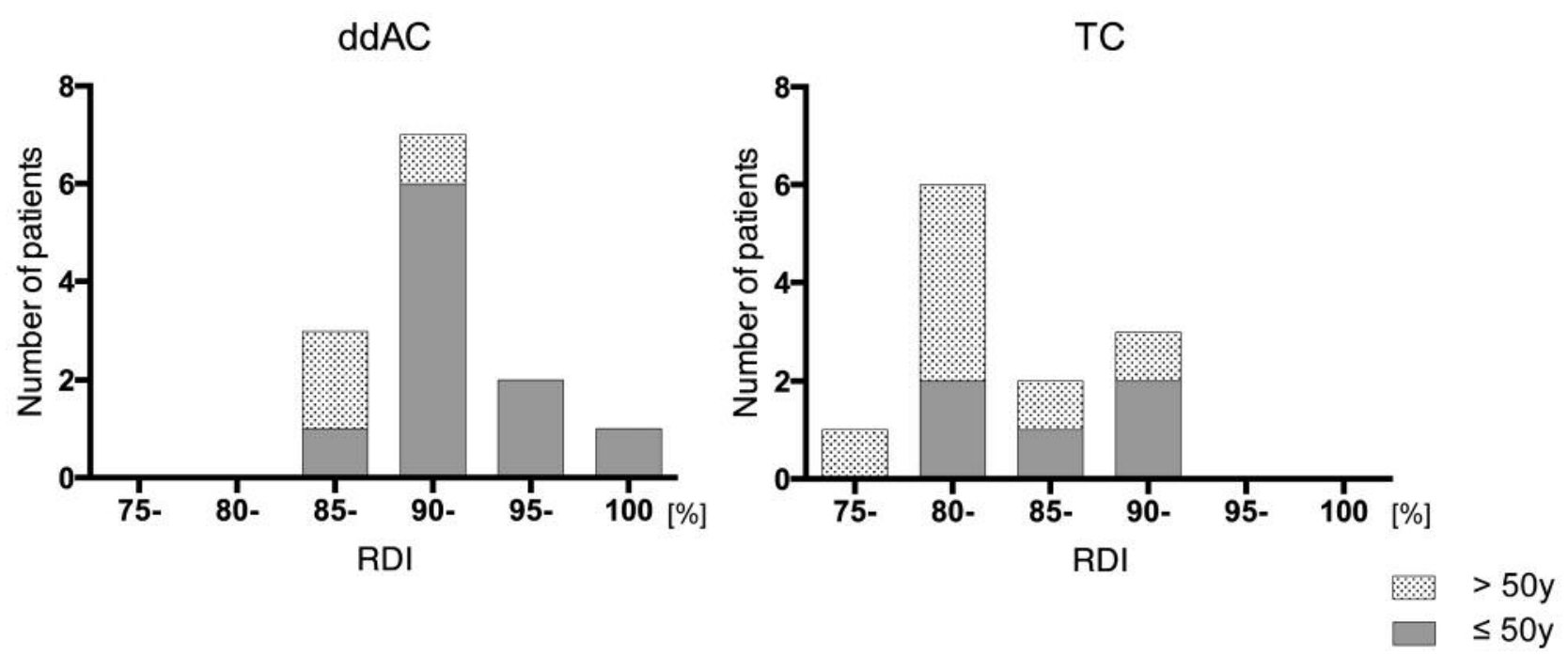

Figure 3. Relative dose intensities (RDI) of the ddAC and TC cases.

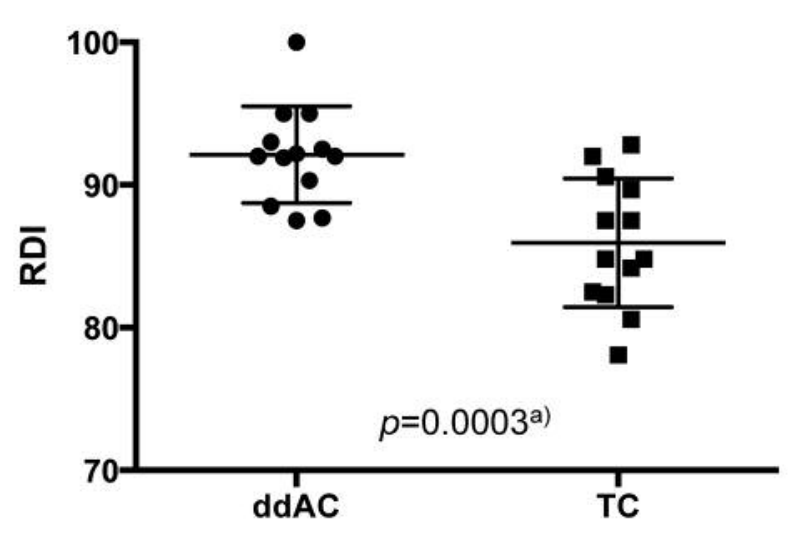

Figure 4. Comparison of the relative dose intensities (RDI) of ddAC and TC. ${ }^{a}$ Logistic regression.

\section{Discussion}

To the best of our knowledge, this is the first study to compare serum G-CSF concentrations while pegfilgrastim (3.6 mg) is subcutaneously administered between bi- or triweekly chemotherapy cycles. Patients in this study never had ANCs <1,500 during any of their treatments. However, there were differences between serum G-CSF concentration and ANC between the bi- and tri-weekly regimens. Peak serum G-CSF concentrations were achieved during the second week in ddAC cases; however, the ANC increased until the end of chemotherapy. After subcutaneous injection, filgrastim is primary eliminated by the kidney and neutrophils or neutrophil precursors; the latter

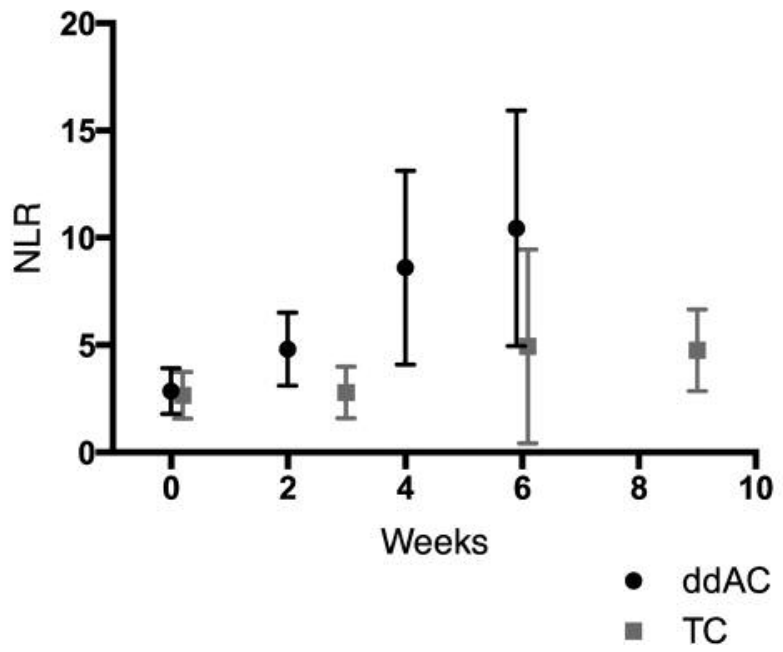

Figure 5. Neutrophil-lymphocyte ratios (NLR).

presumptively involves growth factor-receptor complex formation via endocytosis, and subsequent degradation inside the cells. The PEG-ylation of filgrastim blocks renal clearance; thus, neutrophil-mediated clearance is the predominant elimination pathway for pegfilgrastim (17). In cases of bi-weekly pegfilgrastim administration, there may be insufficient pegfilgrastim metabolism by neutrophil GCSF receptors. It is possible that some component of the G-CSF receptor is refractory when administering pegfilgrastim bi-weekly. Further investigations into the optimal pegfilgrastim administration interval for female Japanese patients is required. 
Meanwhile, in TC cases with pegfilgrastim administration every 3 weeks, changes in both serum G-CSF concentrations and ANCs were more gradual over four treatment cycles than in the ddAC cases. Peak serum G-CSF concentrations were achieved between 6-9 weeks and the ANC decreased slightly during the first 3 weeks, but then gradually increased until the ninth week. Administering pegfilgrastim every 3 weeks may keep an appropriate balance between G-CSF metabolism by the G-CSF receptors of neutrophils and ANC maintenance.

The neutrophil/lymphocyte ratio (NLR) reflects cancer immunity. A systematic review and meta-analysis of 40,559 patients indicated that a high NLR $(>4.0)$ is associated with reduced overall survival in many solid tumours, including breast cancer (hazard ratio $=1.81 ; 95 \%$ confidence interval $=1.67-1.97$; $p<0.001$ ) (18). Additionally, it was reported that NLR was a marker of systematic inflammation that could predict survival and chemotherapeutic outcomes in metastatic colorectal cancer (19). The NLR of both ddAC and TC cases continually increased over our study (Figure 5); however, these reactions are considered temporary because of pegfilgrastim treatment. There was no difference in cancer immunity between patients receiving pegfilgrastim on bi- or tri-weekly schedules.

When neutropenia is observed in patients undergoing chemotherapy, the clinicians are forced to choose dose reduction or interval extension to reduce the risk of $\mathrm{FN}$ or infection. In these cases, there is a possibility that lower RDIs will lead to decreased treatment benefits and poorer outcomes. In our study, approximately $25 \%$ of patients reluctantly experienced dose reductions due to strong fatigue or dysgeusia, which restricted activities of daily life. However, no patients experienced treatment delays or FN with prophylactic pegfilgrastim administration. Thus, primary prophylactic pegfilgrastim administration greatly contributed to maintaining RDI. Although it is difficult to compare the RDI of ddAC to TC because they are different regimens, the RDI of ddAC (bi-weekly) was significantly higher than that of TC (tri-weekly). Therefore, ddAC with pegfilgrastim could be a choice that will allow patients to safely and effectively complete their full chemotherapy cycles without reductions or delays. Our results showed that the mean RDI in patients $>50$-year-old was lower than the younger group. It has been reported that $51 \%$ of elderly patients ( $\geq 70$ years) receiving chemotherapy for breast or gynaecological cancers require G-CSF (20). Thus, age is an important factor for evaluating $\mathrm{FN}$ risk $(3,4)$.

This study had certain limitations; first, the sample size was small because ddAC with pegfilgrastim was not permitted in our institution until the start of 2015. However, worthwhile data were obtained from these small samples. Second, endogenous G-CSF concentrations differ greatly among individuals; thus, G-CSF concentrations were evaluated immediately before chemotherapy as an individual-patient standard (16.5-45.4 pg/ml).
In conclusion, primary prophylactic pegfilgrastim administration (either every 2 weeks for ddAC or every 3 weeks for TC) increased serum G-CSF concentrations and maintained ANCs, both of which are required to undergo chemotherapy. Neutrophils usually decrease after receiving chemotherapy, usually reaching their nadir 2 weeks after therapy begins. However, neutrophil counts were recovered as short as 2 weeks after ddAC with pegfilgrastim and kept increasing through subsequent cycles. ddAC with pegfilgrastim is completely safe for female Japanese patients.

\section{Conflicts of Interest}

There are no conflicts of interest regarding this study.

\section{Acknowledgements}

The Authors thank James P. Mahaffey, PhD, from Edanz Group (www.edanzediting.com/ac) for editing a draft of this manuscript. This work was supported by JSPS KAKENHI Grant-in-Aid for JSPS Research Fellow 18J20301.

\section{References}

1 Smith TJ, Khatcheressian J, Lyman GH, Ozer H, Armitage JO, Balducci L, Bennett CL, Cantor SB, Crawford J, Cross SJ, Demetri G, Desch CE, Pizzo PA, Schiffer CA, Schwartzberg L, Somerfield MR, Somlo G, Wade JC, Wade JL, Winn RJ, Wozniak AJ and Wolff AC: 2006 update of recommendations for the use of white blood cell growth factors: an evidence-based clinical practice guideline. J Clin Oncol 24(19): 3187-3205, 2006.

2 Aapro MS, Bohlius J, Cameron DA, Dal Lago L, Donnelly JP, Kearney N, Lyman GH, Pettengell R, Tjan-Heijnen VC, Walewski J, Weber DC and Zielinski C; European Organisation for Research and Treatment of Cancer: 2010 update of EORTC guidelines for the use of granulocyte-colony stimulating factor to reduce the incidence of chemotherapy-induced febrile neutropenia in adult patients with lymphoproliferative disorders and solid tumours. Eur J Cancer 47(1): 8-32, 2011.

3 https://www.nccn.org/professionals/physician_gls/pdf/myeloid_ growth.pdf, accessed 31 March 2018.

4 http://jsco-cpg.jp/item/30/index.html, in Japanese, accessed 31 March 2018.

5 Molineux G: Pegylation: engineering improved pharmaceuticals for enhanced therapy. Cancer Treat Rev 28: 13-16, 2002.

6 Norton L: Theoretical Concepts and the Emerging Role of Taxanes in Adjuvant Therapy. TheOncologist 6(3): 30-35, 2001.

7 Citron ML, Berry DA, Cirrincione C, Hudis C, Winer EP, Gradishar WJ, Davidson NE, Martino S, Livingston R, Ingle JN, Perez EA, Carpenter J, Hurd D, Holland JF, Smith BL, Sartor CI, Leung EH, Abrams J, Schilsky RL, Muss HB and Norton L: Randomized trial of dose-dense versus conventionally scheduled and sequential versus concurrent combination chemotherapy as postoperative adjuvant treatment of node-positive primary breast cancer: first report of Intergroup Trial C9741/Cancer and Leukemia Group B Trial 9741. J Clin Oncol 21(8): 1431-1439, 2003. 
8 Del Mastro L, De Placido S, Bruzzi P, De Laurentiis M, Boni C, Cavazzini G, Durando A, Turletti A, Nisticò C, Valle E, Garrone O, Puglisi F, Montemurro F, Barni S, Ardizzoni A, Gamucci T, Colantuoni G, Giuliano M, Gravina A, Papaldo P, Bighin C, Bisagni G, Forestieri V and Cognetti F: Fluorouracil and dosedense chemotherapy in adjuvant treatment of patients with earlystage breast cancer: an open-label, $2 \times 2$ factorial, randomised phase 3 trial. The Lancet 385(9980): 1863-1872, 2015.

9 Kosaka Y, Rai Y, Masuda N, Takano T, Saeki T, Nakamura S, Shimazaki R, Ito Y, Tokuda Y and Tamura K: Phase III placebocontrolled, double-blind, randomized trial of pegfilgrastim to reduce the risk of febrile neutropenia in breast cancer patients receiving docetaxel/cyclophosphamide chemotherapy. Support Care Cancer 23: 1137-1143, 2015.

10 https://www.nccn.org/professionals/physician_gls/pdf/breast.pdf, accessed 31 March 2018.

11 http://jbcs.xsrv.jp/guidline/, in Japanese, accessed 31 March 2018.

12 Goldhirsch A, Glick JH, Gelber RD, Coates AS, Thurlimann B, Senn HJ, and Panel Members: Meeting highlights: international expert consensus on the primary therapy of early breast cancer 2005. Ann Oncol 16(10): 1569-1583, 2005.

13 Goldhirsch A, Wood WC, Gelber RD, Coates AS, Thurlimann B, Senn HJ and Panel Members: Progress and promise: highlights of the international expert consensus on the primary therapy of early breast cancer 2007. Ann Oncol 18(7): 11331144, 2007.

14 Goldhirsch A, Ingle JN, Gelber RD, Coates AS, Thurlimann B, Senn HJ and Panel Members: Thresholds for therapies: highlights of the St Gallen International Expert Consensus on the primary therapy of early breast cancer 2009. Ann Oncol 20(8): 1319-1329, 2009.
15 Goldhirsch A, Wood WC, Coates AS, Gelber RD, Thurlimann B, Senn HJ and Panel Members: Strategies for subtypes--dealing with the diversity of breast cancer: highlights of the St. Gallen International Expert Consensus on the Primary Therapy of Early Breast Cancer 2011. Ann Oncol 22(8): 1736-1747, 2011.

$16 \mathrm{http}: / / \mathrm{minds} . j c q h c . o r . j p / \mathrm{n} / \mathrm{med} / 4 / \mathrm{med} 0145 / \mathrm{G} 0000520 / 0001$, in Japanese, accessed 31 March 2018.

17 Yang BB and Kido A: Pharmacokinetics and pharmacodynamics of pegfilgrastim. Clinical Pharmacokinetics 50(5): 295-306, 2011.

18 Templeton AJ, McNamara MG, Seruga B, Vera-Badillo FE, Aneja P, Ocana A, Leibowitz-Amit R, Sonpavde G, Knox JJ, Tran B, Tannock IF and Amir E: Prognostic role of neutrophilto-lymphocyte ratio in solid tumors: a systematic review and meta-analysis. J Natl Cancer Inst 106(6): dju124, 2014.

19 Shibutani M, Maeda K, Nagahara H, Ohtani H, Sakurai K, Yamazoe S, Kimura K, Toyokawa T, Amano R, Kubo N, Tanaka H, Muguruma K, Ohira M and Hirakawa K: Significance of markers of systemic inflammation for predicting survival and chemotherapeutic outcomes and monitoring tumor progression in patients with unresectable metastatic colorectal cancer. Anticancer Res 35: 5037-5046, 2015.

20 Falandry C, Krakowski I, Cure H, Carola E, Soubeyran P, Guerin $\mathrm{O}$ and Freyer G: Granulocyte-Colony-Stimulating Factor in Elderly Patients Receiving Chemotherapy for Breast and Gynaecological Cancers: Results of a French Survey. Anticancer Res 34: 5007-5016, 2014.

Received April 11, 2018

Revised June 5, 2018

Accepted June 6, 2018 